\title{
Phosphoserine Phosphatase Is a Novel Prognostic Biomarker on Chromosome 7 in Colorectal Cancer
}

\author{
KUNIAKI SATO ${ }^{1,2}$, TAKAAKI MASUDA ${ }^{1}$, QINGJIANG HU ${ }^{1}$, TARO TOBO ${ }^{3}$, SHINYA KIDOGAMI ${ }^{1}$, \\ YUSHI OGAWA ${ }^{1}$, TOMOKO SAITO ${ }^{1}$, SHO NAMBARA ${ }^{1}$, HISATERU KOMATSU ${ }^{1}$, HIDENARI HIRATA ${ }^{1}$, \\ SHOTARO SAKIMURA ${ }^{1}$, RYUTARO UCHI ${ }^{2}$, NAOKI HAYASHI ${ }^{1}$, TOMOHIRO IGUCHI ${ }^{1}$, \\ HIDETOSHI EGUCHI ${ }^{1}$, SHUHEI ITO ${ }^{1}$, TAKASHI NAKAGAWA ${ }^{2}$ and KOSHI MIMORI ${ }^{1}$ \\ Departments of ${ }^{1}$ Surgery and ${ }^{3}$ Clinical Laboratory Medicine and Pathology, \\ Kyushu University Beppu Hospital, Oita, Japan; \\ ${ }^{2}$ Department of Otorhinolaryngology, Graduate School of Medical Sciences, \\ Kyushu University, Fukuoka, Japan
}

\begin{abstract}
Background/Aim: Amplification of chromosome $7 p$ $(C h .7 p)$ is common in colorectal cancer $(C R C)$. The aim of this study was to identify potential driver genes on Ch.7p that are overexpressed due to DNA copy number amplification and determine their clinical significance in CRC. Materials and Methods: We identified phosphoserine phosphatase (PSPH) as a potential driver gene using a CRC dataset from The Cancer Genome Atlas (TCGA) using a bioinformatics approach. The expression of PSPH in 124 primary CRCs was examined by quantitative reverse transcription polymerase chain reaction $(P C R)$ and immunohistochemistry. The biological effect of PSPH expression was explored by Gene Set Enrichment Analysis (GSEA) using the TCGA dataset. Results: PSPH was overexpressed in tumor tissues and PSPH positively correlated with depth of invasion and distant metastasis. On multivariate analysis, high PSPH expression was an independent poor prognostic factor. These results were supported by GSEA. Conclusion: PSPH could be a novel prognostic biomarker with malignant potential on Ch.7p in CRC.
\end{abstract}

Colorectal cancer (CRC) is one of the most common types of cancer worldwide and the second most common cause of cancer mortality (1). Relapse of CRC frequently occurs despite multidisciplinary therapy, such as surgical resection, chemotherapy and molecular-targeted therapy (2).

Intra-tumor heterogeneity (ITH) is the existent molecular

Correspondence to: Koshi Mimori, MD, Ph.D., Department of Surgery, Kyushu University Beppu Hospital, 4546 Tsurumihara, Beppu 874-0838, Japan. Tel: +81 977271650, Fax: +81 977271651, e-mail: kmimori@beppu.kyushu-u.ac.jp

Key Words: PSPH, colorectal cancer, intra-tumor heterogeneity, prognostic biomarker. and cellular heterogeneity within a single tumor. ITH likely contributes to the occurrence of drug-resistant cells and increases the chance of therapeutic failure, since the presence of distinct subpopulations of cells with different sensitivities to anti-cancer drugs increases the chance of resistance and recurrence (3). Therefore, it is important to identify oncogenes that exist in tumor cells, as such oncogenes could be promising therapeutic targets despite ITH.

Amplification of chromosome 7 occurs frequently in CRC and colorectal adenoma tissues (4-6). By performing multiregional copy number analysis of CRC tumor tissues, we showed that amplification of the short arm of chromosome 7 (Ch.7p) occurs in all regions of an individual tumor (7). These data suggest that amplification of Ch.7p is a fundamental and predominant event in tumorigenesis of CRC. We hypothesized that $\mathrm{Ch} .7 \mathrm{p}$ harbors driver genes that promote tumorigenesis or tumor progression through gainof-function in CRC.

In this study, we aimed to identify a novel potential driver gene on Ch.7p by a bioinformatics approach and determine the clinical significance of its expression in CRC.

\section{Materials and Methods}

Selection of candidate genes. We obtained the RNA sequencing data and the DNA copy number data of 615 CRC patients in The Cancer Genome Atlas (TCGA) from the Broad Institute's Firehose (http://gdac.broadinstitute.org/runs/stddata__2015_08_21/data/ COADREAD/20150821/). The RNA sequencing data also included expression profiles of 51 paired normal colon samples. Using these data, we extracted the candidate genes from 426 genes on Ch.7p that satisfied the following two criteria. First, the DNA copy number and mRNA expression levels had to be positively correlated with each other. Cut-off of the correlation coefficient was determined as 0.4. Second, the gene in question was overexpressed in tumor tissues compared to normal tissues ( $>2$-fold change). 
Patients and clinical samples. All protocols used in this study were approved by the local ethics review board of Kyushu University. A total of 124 patients with CRC who underwent surgical resection of a primary tumor at Kyushu University Beppu Hospital and the affiliated hospitals between 1992 and 2007 were enrolled in this study. Clinicopathological factors and clinical stage were classified using the tumor-node-metastasis (TNM) system of classification. All patients were treated in accordance with the Japanese Society of Cancer of the Colon and Rectum Guidelines for the Treatment of Colorectal Cancer. Written informed consent was obtained from all patients. Resected tumor tissues and paired normal colon tissues were immediately stored in RNAlater (Ambion, Palo Alto, CA, USA), frozen in liquid nitrogen and kept at $-80^{\circ} \mathrm{C}$ until RNA extraction.

RNA extraction and reverse transcription-quantitative polymerase chain reaction ( $R T-q P C R$ ). Total RNA was isolated from the frozen tissue specimens through use of ISOGEN (Nippon Gene, Tokyo, Japan), according to the manufacture's protocol. Complementary DNA (cDNA) was synthesized from $8 \mu \mathrm{g}$ of total RNA using M-MLV reverse transcriptase (Invitrogen, Carlsbad, CA, USA). qPCR was performed using a LightCycler 480 and a SYBR Green I Master kit (Roche Applied Science, Basel, Switzerland) following the manufacturer's protocol. Gene expression was quantified using the following specific oligonucleotide primers: phosphoserine phosphatase $(P S P H)$ : 5'-GCAGTGCCTTTCAAAGCTGC-3' (sense) and 5'TAGGCGACTTACCAGCTCCC-3' (antisense); glyceraldehyde-3phosphate dehydrogenase (GAPDH): 5'-AGCCACATCGCTCAGAC AC-3' (sense) and 5'-GCCCAATACGACCAAATCC-3' (antisense). The expression levels were normalized to the expression of $G A P D H$ as an internal control in each sample. The expression levels were calculated as values relative to the expression level of Human Universal Reference Total RNA (Clontech Laboratories, Palo Alto, CA, USA).

Immunohistochemical analysis. Immunohistochemistry of PSPH in CRC cases was performed on formalin-fixed, paraffin-embedded tissues as previously described (8). A polyclonal rabbit anti-PSPH antibody (HPA020376; Sigma-Aldrich, St. Louis, MO, USA) was used as the primary antibody diluted at 1:200. Tumor histology was independently reviewed by an experienced pathologist.

Gene set enrichment analysis (GSEA). Using the RNA sequencing data of CRC tissues in TCGA, we performed GSEA (9) to elucidate the correlation between the candidate genes and gene sets that are associated with tumor progression.

Statistical analysis. Associations between the variables were tested by the Mann-Whitney $U$-test or Fisher's exact test. Overall survival (OS) curves were plotted according to the Kaplan-Meier method and compared using the log-rank test. Univariate and multivariate analyses were performed using the Cox proportional hazards model to identify independent variables predictive of OS. Statistical analyses were performed using JMP Pro 11 software (SAS Institute, Cary, NC, USA) and R version 3.2.0 (The R Foundation for Statistical Computing, Vienna, Austria). The differences were considered significant when the $p$-value was lower than 0.05 .

\section{Results}

PSPH was identified as a potential driver gene in CRC. We identified $P S P H$, the gene for the rate-limiting enzyme in the serine biosynthesis pathway (SSP), as a potential driver gene on Ch.7p in CRC (Figure 1a). In this pathway, PSPH converts 3-phosphoserine to L-serine, which fuels glycine biosynthesis (10). Glycine is a major source of glutathione (GSH) and purine nucleotides (10). In the TCGA dataset, PSPH mRNA expression in CRC tumor tissues was 2.64fold higher than in normal tissues $(p<0.001)$ (Figure 1b). $P S P H$ mRNA expression and $P S P H$ copy number were positively correlated $(\mathrm{R}=0.51, p<0.001)$. Furthermore, copy number amplification of $P S P H$ was observed in most of the CRC tissues in the TCGA dataset (Figure 1c).

PSPH was overexpressed in CRC. Next, we conducted qRTPCR to measure the expression levels of $P S P H$ mRNA in tumor tissues and paired normal colon tissues from $124 \mathrm{CRC}$ patients from our Hospital. Consistent with the result from the TCGA dataset, PSPH mRNA expression was significantly higher in CRC tissues than in normal colon tissues $(p<0.001)$ (Figure 2a). We also performed immunohistochemical staining to observe PSPH protein expression in CRC tissues and normal colon tissues. PSPH was strongly stained in tumor cells, whereas it was weak to moderate in normal colon cells (Figure 2b). Higher magnification of the image revealed cytoplasmic staining of PSPH in tumor cells.

Prognostic significance of PSPH mRNA expression in patients with $C R C$. We divided the 124 patients with CRC into a high PSPH mRNA expression group $(\mathrm{n}=57)$ and a low $P S P H$ mRNA expression group $(\mathrm{n}=67)$ using the minimum $p$-value approach (11). The high PSPH mRNA expression group had poorer OS than the low expression group in our dataset (Figure 2c, left). Furthermore, we found that the high PSPH mRNA expression group had poorer prognosis than the low expression group in the TCGA dataset (Figure 2c, right). Multivariate analysis demonstrated that high $P S P H$ mRNA expression was an independent prognostic factor in our dataset (5-year survival rate; low/high: 89.6/66.7\%, $p<0.01$ ) (Table I).

Clinicopathological characteristics of PSPH mRNA expression in patients with CRC. Clinicopathological analysis of our dataset revealed that high expression of PSPH mRNA was correlated with depth of invasion $(p<0.05)$ and distant metastasis $(p<0.05)$ (Table II).

Correlation between PSPH mRNA expression and gene sets associated with cell-cycle progression and apoptosis in CRC. GSEA revealed a positive correlation between $P S P H$ mRNA expression and the expression of a gene set involved in cellcycle progression $(p<0.05)$ (Figure 3a, left). Negative correlation between PSPH mRNA expression and the expression of a gene set up-regulated in apoptosis progression was also detected $(p<0.05)$ (Figure 3a, right). 
$\mathrm{a}$

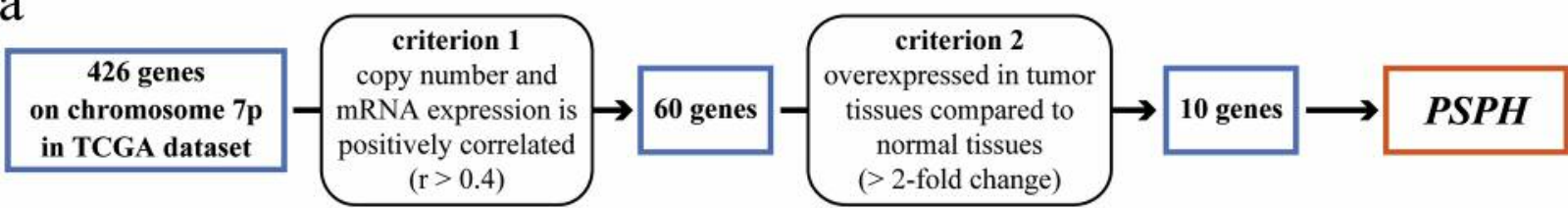

$\mathrm{b}$

C
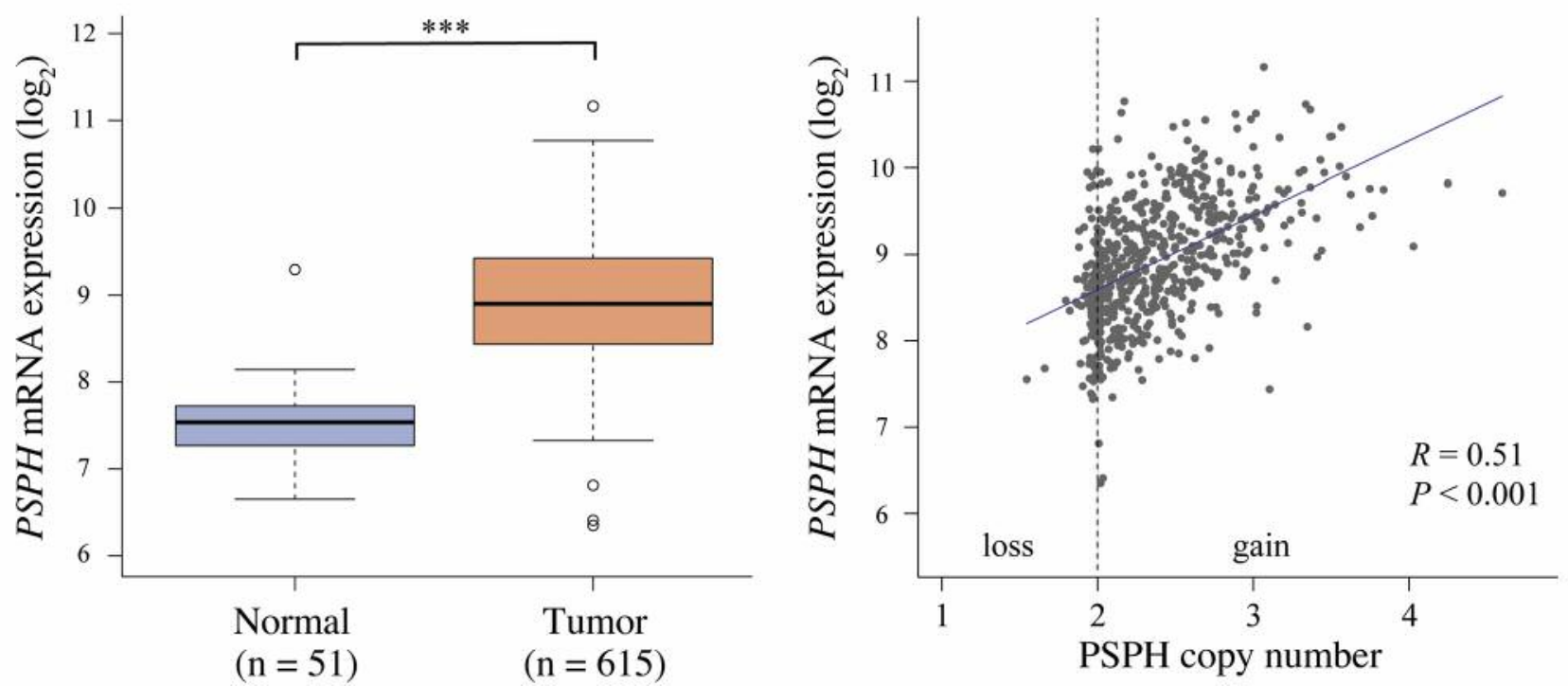

Figure 1. Identification of candidate driver genes on chromosome $7 p$ in CRC. (a) Schematic diagram of the strategy for candidate genes selection as described in Materials and Methods. (b) Boxplot of PSPH mRNA expression in 615 CRC tissues and 51 normal colon tissues in the TCGA dataset. (c) Correlation between PSPH copy number and PSPH mRNA expression in the TCGA dataset. $R$ is the Pearson correlation coefficient. ***p<0.001.

Table I. Univariate and multivariate analyses of clinicopathological factors affecting 5-year overall survival.

\begin{tabular}{|c|c|c|c|c|c|c|}
\hline \multirow[t]{2}{*}{ Factor } & \multicolumn{3}{|c|}{ Univariate analysis } & \multicolumn{3}{|c|}{ Multivariate analysis } \\
\hline & HR & $95 \% \mathrm{CI}$ & $p$-Value & HR & $95 \% \mathrm{CI}$ & $p$-Value \\
\hline Age $(<65 / \geq 65)$ & 2.76 & $1.27-6.09$ & $0.011 *$ & 1.84 & $0.80-4.38$ & 0.152 \\
\hline Gender (male/female) & 0.99 & $0.45-2.33$ & 0.989 & & & \\
\hline Histology (poorly/well or moderate) & 5.13 & $1.49-13.5$ & $0.013^{*}$ & 7.33 & $1.71-26.9$ & $0.010 *$ \\
\hline Tumor size $(\geq 3 \mathrm{~cm} /<3 \mathrm{~cm})$ & 7.65 & $1.62-137$ & $0.005^{*}$ & 2.65 & $0.45-51.1$ & 0.319 \\
\hline Depth of invasion (SS, SE, SI/ M, SM, MP) & 12.9 & $2.73-230$ & $<0.001 *$ & 2.39 & $0.36-48.5$ & 0.406 \\
\hline Lymph node metastasis (present/absent) & 4.41 & $1.87-12.1$ & $<0.001 *$ & 2.67 & $1.07-7.74$ & $0.035^{*}$ \\
\hline Distant metastasis (present/absent) & 8.44 & $0.43-59.5$ & 0.129 & & & \\
\hline Venous invasion (present/absent) & 3.81 & $1.74-8.32$ & $0.001 *$ & 2.44 & $1.00-6.11$ & $0.049 *$ \\
\hline Lymphatic invasion (present/absent) & 2.08 & $0.96-4.65$ & 0.064 & & & \\
\hline$P S P H$ expression (high/low) & 3.81 & $1.67-9.78$ & $0.001 *$ & 4.66 & $1.69-15.7$ & $0.002 *$ \\
\hline
\end{tabular}

M, Mucosa; SM, submucosa; MP, muscular propria; SS, subserosa; SE, serosal invasion; SI, invasion to adjacent organs; HR, hazard ratio; CI, confidence interval; PSPH, phosphoserine phosphatase. *Statistically significant.

\section{Discussion}

Here, we identified $P S P H$ as a candidate of driver gene on Ch.7p in CRC. We demonstrated that overexpression of
PSPH was associated with depth of invasion and distant metastasis and, also, that it was an independent poor prognostic factor in CRC patients. Moreover, overexpression of $P S P H$ was likely caused by amplification of Ch.7p. These 


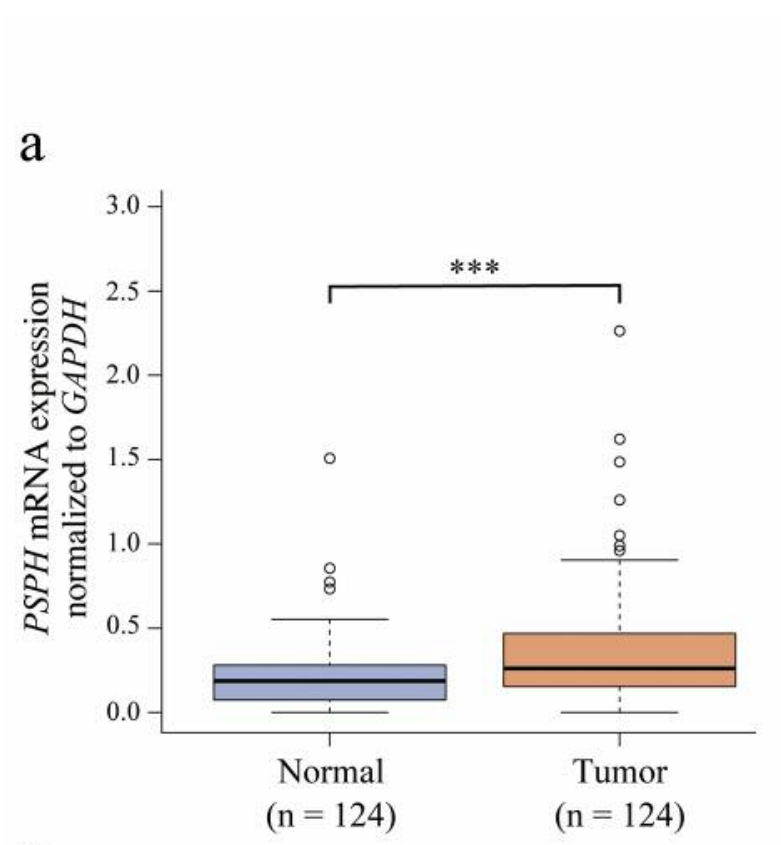

b

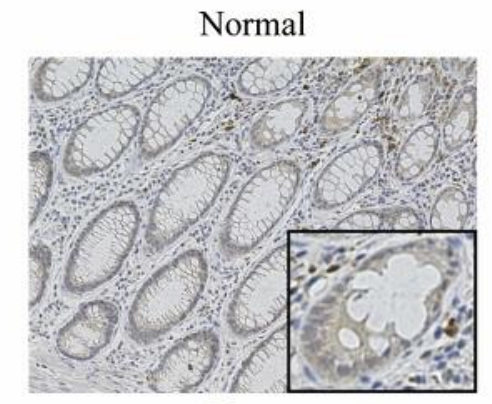

\section{Tumor}

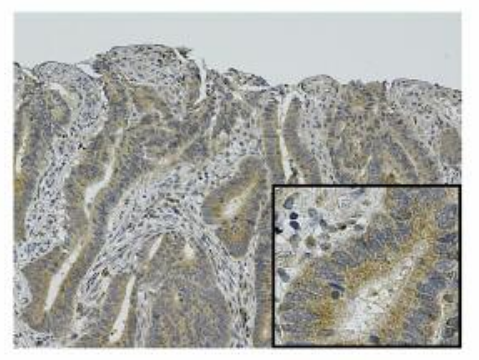

C
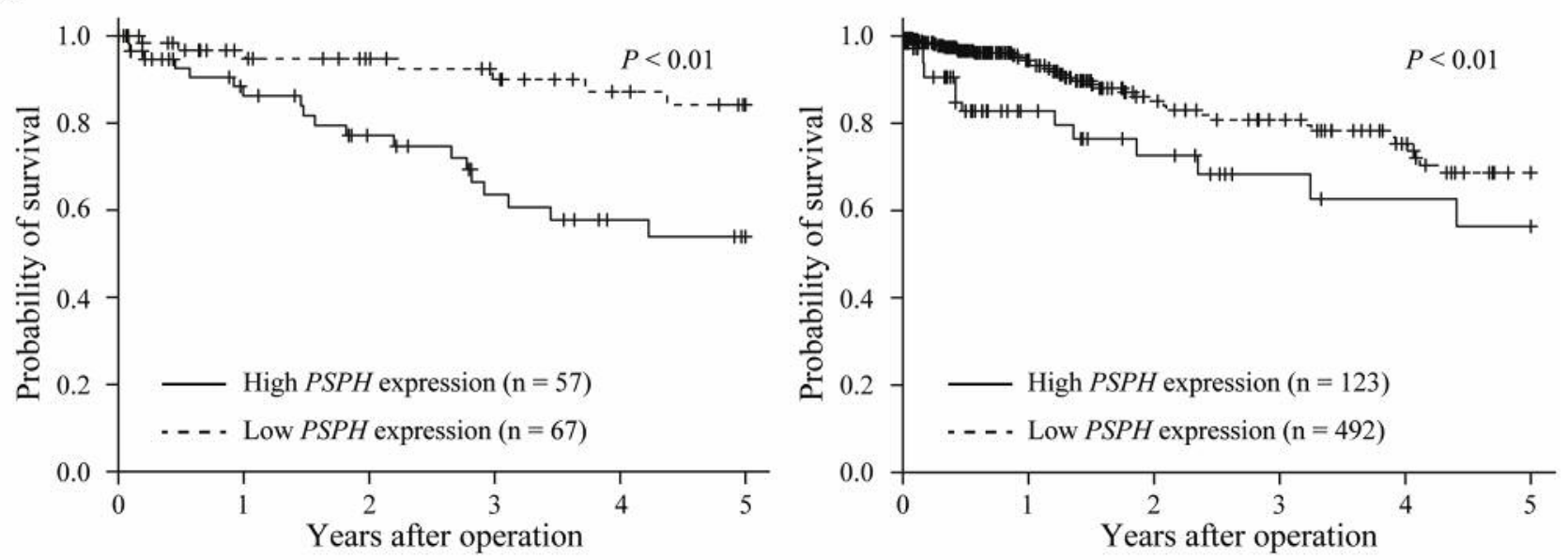

Figure 2. Clinical significance of PSPH expression in CRC. (a) PSPH mRNA expression in 124 CRC tissues and paired normal colon tissues by qRT-PCR. (b) Immunohistochemical staining for PSPH in normal colon tissues (left) and CRC tissues (right). (c) Kaplan-Meier overall survival curves for patients with CRC according to PSPH mRNA expression in two independent datasets. (left; Kyushu University Beppu Hospital, $n=124$ ) (right; TCGA dataset, $n=615$ ). Original magnification; $\times 200 . * * *<<0.001$.

findings indicate that $P S P H$ expression is a novel biomarker for poor prognosis and could play an important role in tumor progression of CRC.

As shown in Figure 3b, PSPH plays a crucial role in the production of L-serine in SSP (10). L-serine is converted to glycine, a major source of GSH and purine nucleotides (10). Importantly, glycine consumption and activation of mitochondrial glycine biosynthesis correlate with rapid proliferation across several types of cancer cell lines, while starvation of glycine inhibits proliferation by prolonging the $\mathrm{G}_{1}$ phase of the cell cycle (12). It is reported that the accumulation of GSH increases the ability to scavenge reactive oxygen species (ROS), which results in promoting proliferation of cancer cells and resistance against druginduced apoptosis $(13,14)$. Consistent with these data, our GSEA with the TCGA dataset indicated that high PSPH expression is significantly associated with cell cycle progression and acquisition of an anti-apoptotic characteristic of CRC. Moreover, SSP enzymes, such as PSPH, are overexpressed in several types of cancer (15-18) and overexpression of SSP enzymes has been associated with poor prognosis (18-22). These data support our hypothesis 
a

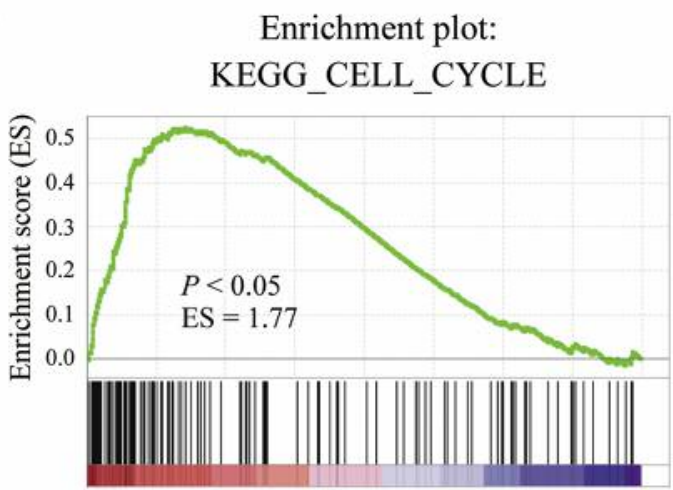

Enrichment plot: HALLMARK_APOPTOSIS

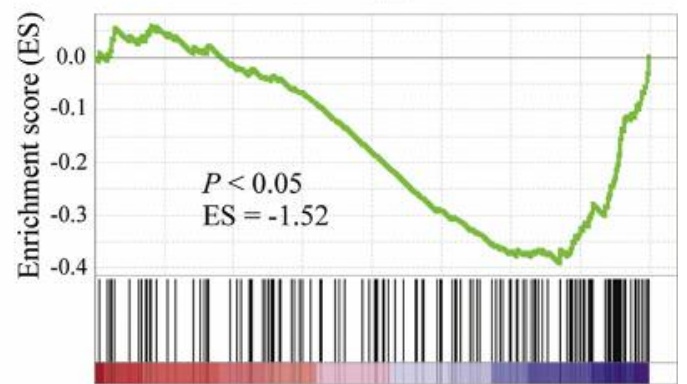

b

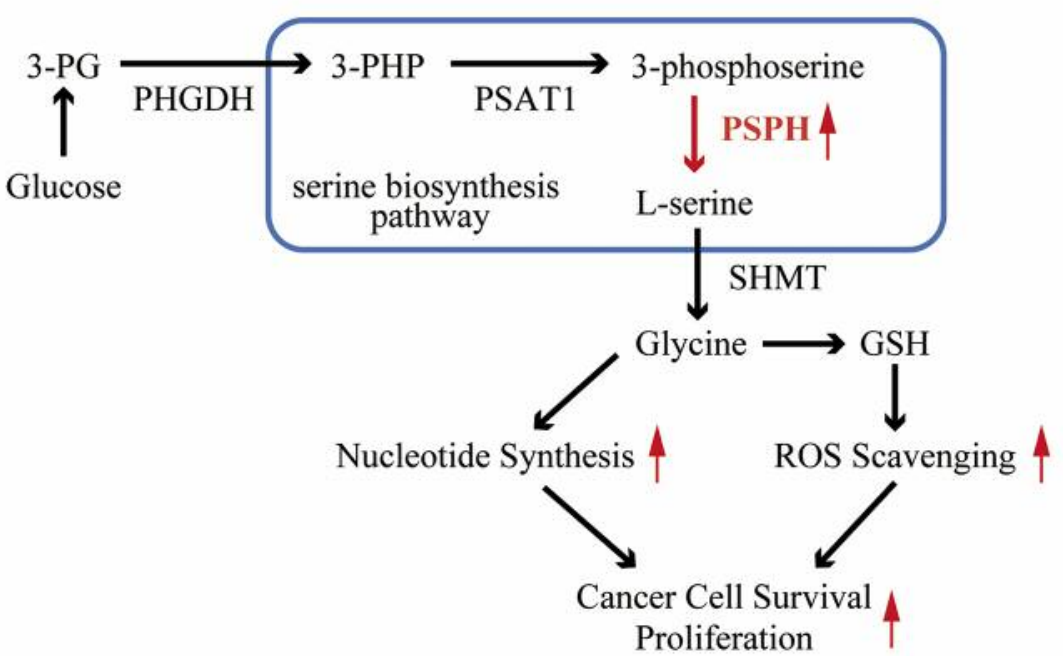

Figure 3. GSEA for PSPH mRNA expression in the TCGA dataset. (a) GSEA using the TCGA dataset showed that PSPH mRNA expression was positively correlated with the expression of a gene set involved in cell cycle progression (left), whereas negatively correlated with a gene set upregulated in apoptosis progression (right). (b) Schematic model indicating that overexpression of PSPH promotes proliferation and cancer cell survival. ES, Enrichment score; 3-PG, 3-phosphoglycerate; 3-PHP, 3-phosphohydroxy pyruvate; PHGDH, phosphoglycerate; PSAT1, phosphoserine aminotransferase-1; PSPH, phosphoserine phosphatase; SHMT, serine hydroxymethyltransferase; GSH, glutathione; ROS, reactive oxygen species.

that overexpression of PSPH due to amplification of $\mathrm{Ch} .7 \mathrm{p}$ enhances its function in serine synthesis and accelerates the production of glycine and GSH, which leads to an aggressive phenotype of tumor cells and poor prognosis in CRC patients.

Furthermore, knockdown of PSPH reduces GSH and impairs anti-oxidant activity of CRC cell lines, thereby enhancing the anticancer efficacy of 5-fluorouracil (5-FU) in vitro and in vivo (14). Of note, preclinical studies are ongoing for small molecules targeting SSP enzymes in the treatment of cancer (23). These reports strongly support our clinical findings that overexpression of $P S P H$ is associated with tumor aggressiveness and poor prognosis in CRC.

In conclusion, we found that PSPH copy number amplification accompanies alterations in Ch.7p in CRC. Overexpression of $P S P H$ is likely a ubiquitous driving event in all CRC cells. Thus, $P S P H$ expression constitutes a novel prognostic biomarker for the malignant potential of CRC. PSPH may offer a therapeutic target to overcome ITH in CRC patients.

\section{Acknowledgements}

This research used the super-computing resource provided by the Human Genome Center, The Institute of Medical Science, The University of Tokyo (http://sc.hgc.jp/shirokane.html). We thank K. Oda, M. Kasagi, M. Sakuma, M. Oshiumi, N. Mishima and T. Kawano for their technical assistance. This work was supported in part by the following grants and foundations: Japan Society for the Promotion of Science Grant-in-Aid for Scientific Research (grant number 16K10543, 16H01576, 16K07177, 16K10397, 16K19197 and 16K19107) and the OITA Cancer Research Foundation. 
Table II. PSPH mRNA expression and clinicopathological factors in patients with CRC $(n=124)$.

\begin{tabular}{|c|c|c|c|}
\hline Factor & $\begin{array}{l}\text { High } \\
(\mathrm{n}=57)\end{array}$ & $\begin{array}{c}\text { Low } \\
(\mathrm{n}=67)\end{array}$ & $p$-Value \\
\hline Age & & & 0.254 \\
\hline$<65$ & 22 & 19 & \\
\hline$\geq 65$ & 35 & 48 & \\
\hline Gender & & & 0.344 \\
\hline Male & 35 & 47 & \\
\hline Female & 22 & 20 & \\
\hline Maximum tumor size & & & 0.354 \\
\hline$<3 \mathrm{~cm}$ & 49 & 53 & \\
\hline$\geq 3 \mathrm{~cm}$ & 8 & 14 & \\
\hline UICC TNM stage & & & 0.857 \\
\hline $\mathrm{I} / \mathrm{II}$ & 28 & 35 & \\
\hline III/IV & 29 & 32 & \\
\hline Depth of invasion & & & $0.017 *$ \\
\hline M, SM, MP & 10 & 25 & \\
\hline SS, SE, SI & 47 & 42 & \\
\hline Lymph node metastasis & & & 1.000 \\
\hline Absent & 28 & 34 & \\
\hline Present & 29 & 33 & \\
\hline Venous invasion & & & 0.062 \\
\hline Absent & 38 & 55 & \\
\hline Present & 19 & 12 & \\
\hline Lymphatic invasion & & & 0.149 \\
\hline Absent & 27 & 41 & \\
\hline Present & 30 & 26 & \\
\hline Distant metastasis & & & $0.042 *$ \\
\hline Absent & 53 & 67 & \\
\hline Present & 4 & 0 & \\
\hline Histology & & & 1.000 \\
\hline Well or moderately differentiated & 54 & 63 & \\
\hline Poorly differentiated & 3 & 4 & \\
\hline
\end{tabular}

CRC, Colorectal cancer; PSPH, phosphoserine phosphatase; M, mucosa; SM, submucosa; MP, muscular propria; SS, subserosa; SE, serosal invasion; SI, invasion to adjacent organs; UICC TNM stage, Union for International Cancer Control tumor-node-metastasis stage. *Statistically significant;

\section{Disclosure}

None of the Authors has a conflict of interest to disclose.

\section{References}

1 Jemal A, Bray F, Center MM, Ferlay J, Ward E and Forman D: Global cancer statistics. CA Cancer J Clin 61: 69-90, 2011.

2 Markowitz SD and Bertagnolli MM: Molecular basis of colorectal cancer. New Engl J Med 361: 2449-2460, 2009.

3 McGranahan $\mathrm{N}$ and Swanton C: Biological and therapeutic impact of intratumor heterogeneity in cancer evolution. Cancer Cell 27: 15-26, 2015.

4 Douglas EJ, Fiegler H, Rowan A, Halford S, Bicknell DC, Bodmer W, Tomlinson IP and Carter NP: Array comparative genomic hybridization analysis of colorectal cancer cell lines and primary carcinomas. Cancer Res 64: 4817-4825, 2004.

5 Herbergs J, Arends JW, Bongers EM, Ramaekers FC and Hopman AH: Clonal origin of trisomy for chromosome 7 in the epithelial compartment of colon neoplasia. Gene Chromosome Canc 16: 106-112, 1996.

6 Zarzour P, Boelen L, Luciani F, Beck D, Sakthianandeswaren A, Mouradov D, Sieber OM, Hawkins NJ, Hesson LB, Ward RL and Wong JW: Single nucleotide polymorphism array profiling identifies distinct chromosomal aberration patterns across colorectal adenomas and carcinomas. Gene Chromosome Canc 54: 303-314, 2015.

7 Uchi R, Takahashi Y, Niida A, Shimamura T, Hirata H, Sugimachi K, Sawada G, Iwaya T, Kurashige J, Shinden Y, Iguchi T, Eguchi H, Chiba K, Shiraishi Y, Nagae G, Yoshida K, Nagata Y, Haeno H, Yamamoto H, Ishii H, Doki Y, Iinuma H, Sasaki S, Nagayama S, Yamada K, Yachida S, Kato M, Shibata T, Oki E, Saeki H, Shirabe K, Oda Y, Maehara Y, Komune S, Mori M, Suzuki Y, Yamamoto K, Aburatani H, Ogawa S, Miyano $\mathrm{S}$ and Mimori K: Integrated multiregional analysis proposing a new model of colorectal cancer evolution. PLoS Genet 12: e1005778, 2016.

8 Ueda M, Iguchi T, Nambara S, Saito T, Komatsu H, Sakimura S, Hirata H, Uchi R, Takano Y, Shinden Y, Eguchi H, Masuda T, Sugimachi K, Yamamoto H, Doki Y, Mori M and Mimori K: Overexpression of Transcription termination factor 1 is associated with a poor prognosis in patients with colorectal cancer. Ann Surg Oncol 22(Suppl 3): S1490-S1498, 2015.

9 Subramanian A, Tamayo P, Mootha VK, Mukherjee S, Ebert BL, Gillette MA, Paulovich A, Pomeroy SL, Golub TR, Lander ES and Mesirov JP: Gene set enrichment analysis: a knowledgebased approach for interpreting genome-wide expression profiles. Proc Natl Acad Sci USA 102: 15545-15550, 2005.

10 Amelio I, Cutruzzola F, Antonov A, Agostini M and Melino G: Serine and glycine metabolism in cancer. Trends Biochem Sci 39: 191-198, 2014.

11 Mizuno H, Kitada K, Nakai K and Sarai A: PrognoScan: A new database for meta-analysis of the prognostic value of genes. BMC Med Genomics 2: 18, 2009.

12 Jain M, Nilsson R, Sharma S, Madhusudhan N, Kitami T, Souza AL, Kafri R, Kirschner MW, Clish CB and Mootha VK: Metabolite profiling identifies a key role for glycine in rapid cancer cell proliferation. Science 336: 1040-1044, 2012.

13 Trachootham D, Alexandre J and Huang P: Targeting cancer cells by ROS-mediated mechanisms: A radical therapeutic approach? Nat Rev Drug Discov 8: 579-591, 2009.

$14 \mathrm{Li} \mathrm{X}$, Xun Z and Yang Y: Inhibition of phosphoserine phosphatase enhances the anticancer efficacy of 5-fluorouracil in colorectal cancer. Biochem Biophys Res Commun 477: 633639, 2016.

15 Sun L, Song L, Wan Q, Wu G, Li X, Wang Y, Wang J, Liu Z, Zhong X, He X, Shen S, Pan X, Li A, Wang Y, Gao P, Tang H and Zhang $\mathrm{H}$ : cMyc-mediated activation of serine biosynthesis pathway is critical for cancer progression under nutrient deprivation conditions. Cell Res 25: 429-444, 2015.

16 DeNicola GM, Chen PH, Mullarky E, Sudderth JA, Hu Z, Wu D, Tang H, Xie Y, Asara JM, Huffman KE, Wistuba, II, Minna JD, DeBerardinis RJ and Cantley LC: NRF2 regulates serine biosynthesis in non-small cell lung cancer. Nat Genet 47: 14751481, 2015. 
17 Yoon S, Kim JG, Seo AN, Park SY, Kim HJ, Park JS, Choi GS, Jeong JY, Jun do Y, Yoon GS and Kang BW: Clinical implication of serine metabolism-associated enzymes in colon cancer. Oncology 89: 351-359, 2015.

18 Xian Y, Zhang S, Wang X, Qin J, Wang W and Wu H: Phosphoglycerate dehydrogenase is a novel predictor for poor prognosis in gastric cancer. Onco Targets Ther 9: 5553-5560, 2016.

19 Antonov A, Agostini M, Morello M, Minieri M, Melino G and Amelio I: Bioinformatics analysis of the serine and glycine pathway in cancer cells. Oncotarget 5: 11004-11013, 2014.

20 Liao KM, Chao TB, Tian YF, Lin CY, Lee SW, Chuang HY, Chan TC, Chen TJ, Hsing $\mathrm{CH}$, Sheu MJ and $\mathrm{Li} \mathrm{CF}$ : Overexpression of the PSAT1 gene in nasopharyngeal carcinoma is an indicator of poor prognosis. J Cancer 7: 1088-1094, 2016.

21 Samanta D, Park Y, Andrabi SA, Shelton LM, Gilkes DM and Semenza GL: PHGDH expression is required for mitochondrial redox homeostasis, breast cancer stem cell maintenance, and lung metastasis. Cancer Res 76: 4430-4442, 2016.
22 Kim HM, Jung WH and Koo JS: Site-specific metabolic phenotypes in metastatic breast cancer. J Transl Med 12: 354, 2014.

23 Mullarky E, Lucki NC, Beheshti Zavareh R, Anglin JL, Gomes AP, Nicolay BN, Wong JC, Christen S, Takahashi H, Singh PK, Blenis J, Warren JD, Fendt SM, Asara JM, DeNicola GM, Lyssiotis CA, Lairson LL and Cantley LC: Identification of a small molecule inhibitor of 3-phosphoglycerate dehydrogenase to target serine biosynthesis in cancers. Proc Natl Acad Sci USA 113: 1778-1783, 2016.
Received March 15, 2017

Revised March 28, 2017

Accepted March 31, 2017 